\title{
Study on a Spinorial Representation of Linear Canonical Transformation
}

\author{
Raoelina Andriambololona, Ravo Tokiniaina Ranaivoson, Hanitriarivo Rakotoson \\ Information Technology and Theoretical Physics Department, National Institute of Nuclear Science and Techniques (INSTN-Madagascar), \\ Antananarivo, Madagascar
}

\section{Email address:}

raoelina.andriambololona@gmail.com (R. Andriambololona), raoelinasp@yahoo.fr (R. Andriambololona), jacquelineraoelina@hotmail.com (R. Andriambololona), tokhiniaina@gmail.com (R. T. Ranaivoson), tokiniainaravor13@gmail.com (R. T. Ranaivoson),infotsara@gmail.com(H. Rakotoson)

\section{To cite this article:}

Raoelina Andriambololona, Ravo Tokiniaina Ranaivoson, Hanitriarivo Rakotoson. Study on a Spinorial Representation of Linear Canonical Transformation. International Journal of Applied Mathematics and Theoretical Physics. Vol. 5, No. 3, 2019, pp. 58-65. doi: $10.11648 /$ j.jjamtp.20190503.12

Received: June 15, 2019; Accepted: July 30, 2019; Published: August 26, 2019

\begin{abstract}
This paper is mainly focused on the description of an approach for establishing a spinorial representation of linear canonical transformations. It can be considered as a continuation of our previous works concerning linear canonical transformations and phase space representation of quantum theory. The said method is based on the development of an adequate parameterization of linear canonical transformations which permits to represent them with special pseudo-orthogonal transformations. Obtaining this pseudo-orthogonal representation makes it possible to establish the spinorial representation exploiting the well-known relation existing between special pseudo-orthogonal and spin groups. The cases of one dimension and general multidimensional theories are both studied. The design of the pseudo-orthogonal transformation associated to a linear canonical transformation is achieved by introducing adequate operators which are linear combinations of reduced momentum and coordinate operators. It is shown that a linear canonical transformation is equivalent to a special pseudoorthogonal transformation defined in the set formed by these adequate operators. The spinorial representation is then deduced by defining a composite operator which is linear combinations of the tensorial products of the generators of the Clifford algebra with the adequate operators defining the special pseudo-orthogonal representation. It is established that unlike the case of a spinorial representation associated with an ordinary commutative vector space, the main invariant corresponding to the transformation is not the square of the composite operator but a higher degree polynomial function of it.
\end{abstract}

Keywords: Linear Canonical Transformation, Special Pseudo-Orthogonal Transformation, Clifford Algebra, Spin Group, Spinorial Representation, Quantum Theory

\section{Introduction}

In our previous papers [1-4], we have performed a series of study on a phase space representation of quantum theory and Linear Canonical Transformations (LCTs). LCTs have already been studied in various contexts [5-9] but our work is focused on their study in the framework of quantum theory. In the paper [2], we have established that there is an isomorphism between the dispersion operator algebra and the Lie algebra $\mathfrak{s p}\left(2 \mathrm{~N}_{+}, 2 \mathrm{~N}_{-}\right)$of the Lie group $\operatorname{Sp}\left(2 \mathrm{~N}_{+}, 2 \mathrm{~N}_{-}\right)$corresponding to the set of LCTs associated to a pseudo-euclidian space of signature $\left(\mathrm{N}_{+}, \mathrm{N}_{-}\right)$. This isomorphism permits to establish an unitary representation of LCTs. In this paper, our main goal is to describe a method for the establishment of a spinorial representation of LCTs. The approach consists mainly of associating a special pseudo-orthogonal transformation to a LCT. Then, the well-known relations existing between special pseudo-orthogonal and spin groups [10-12] are exploited to establish the spinorial representation of LCTs.

In the work [2], we have introduced operators defined from the momentum $p$ and coordinate operators $x$ of a particle. Some of these operators will be used throughout the present paper. These operators are the reduced operators $\not$ and $\mathcal{*}$, the reduced dispersions operators $\beth^{+}, \beth^{-}$and $\beth^{\times}$and their multidimensional generalization $p_{\mu}, \mathcal{x}_{\mu}, \beth_{\mu \nu}^{+}, \beth_{\mu \nu}^{-}$and $\beth_{\mu \nu}^{\times}$. The notations that we use throughout this paper are inspired from [13]. Operators defined from the momentum and coordinate operators are written in bold. 


\section{Special Pseudo-Orthogonal Transformation Associated to a LCT}

\subsection{Case of One Dimension Theory}

In the framework of one dimension quantum mechanics, a LCT is a linear transformation of the form [2]

$$
\left\{\begin{array}{l}
\not^{\prime}=\Pi \not p+\Theta \mathscr{X} \\
\mathscr{X}^{\prime}=\Xi p+\Lambda \mathscr{X}
\end{array} \Leftrightarrow\left(\begin{array}{ll}
\not^{\prime} & \mathscr{X}^{\prime}
\end{array}\right)=\left(\begin{array}{ll}
p & \mathscr{X}
\end{array}\right)\left(\begin{array}{ll}
\Pi & \Xi \\
\Theta & \Lambda
\end{array}\right)\right.
$$

which leaves invariant the canonical commutation relation: $\left[x^{\prime}, p^{\prime}\right]=[x, p]=i$. The consequence of this condition is that the matrix $g=\left(\begin{array}{ll}\Pi & \Xi \\ \Theta & \Lambda\end{array}\right)$ which describes the transformation must have a determinant equal to $1 . \mathrm{g}$ belongs to the Special Linear group $S L(2)$. The Lie algebra $\mathfrak{s l}(2)$ of the Lie group $S L(2)$ is the set of $2 \times 2$ square matrices with trace equal to zero. We choose the parameterization

$$
g=\left(\begin{array}{cc}
\Pi & \Xi \\
\Theta & \Lambda
\end{array}\right)=\exp \left[\left(\begin{array}{cc}
\mu & \varphi-\theta \\
\varphi+\theta & -\mu
\end{array}\right)\right]
$$

To establish a spinorial representation, we have to find a special pseudo-orthogonal representation corresponding to a LCT. We define the following operators

$$
\left\{\begin{array}{l}
p^{+}=\frac{1}{\sqrt{2}}\left(\sigma^{1} \otimes p+\sigma^{2} \otimes x\right) \\
\mathfrak{x}^{-}=\frac{1}{\sqrt{2}}\left(\sigma^{1} \otimes \not{x}-\sigma^{2} \otimes p\right) \\
\mathfrak{x}^{+}=\frac{1}{\sqrt{2}}\left(\sigma^{1} \otimes \not{x}+\sigma^{2} \otimes p\right) \\
p^{-}=\frac{1}{\sqrt{2}}\left(\sigma^{1} \otimes p-\sigma^{2} \otimes x\right)
\end{array}\right.
$$

in which $\sigma^{1}$ and $\sigma^{2}$ are the generators of the Clifford algebra $\mathfrak{C}(2,0)=\mathfrak{C}(2)$ (for instance the Pauli matrices). From the relations (1), (2) and (3) we deduce that for an infinitesimal LCT, the laws of transformations of $p^{+}, x^{-}, x^{+}$and $p^{-}$are

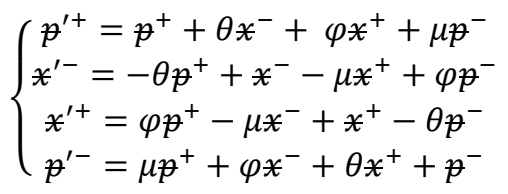

The infinitesimal transformation (4) can be put in the matricial form

$$
\left(\not^{\prime+} \quad \mathscr{x}^{\prime-} \quad \mathscr{X}^{\prime+} \quad p^{\prime-}\right)=\left(\begin{array}{llll}
\not^{+} & \mathscr{X}^{-} & \mathscr{X}^{+} & p^{-}
\end{array}\right)\left(I_{4}+\mathcal{X}\right)
$$

in which $I_{4}$ is the $4 \times 4$ identity matrix and $X$ is the $4 \times 4$ matrix

$$
\chi=\left(\begin{array}{cccc}
0 & -\theta & \varphi & \mu \\
\theta & 0 & -\mu & \varphi \\
\varphi & -\mu & 0 & \theta \\
\mu & \varphi & -\theta & 0
\end{array}\right)
$$

It is easy to verify that $\mathcal{X}$ belongs to the Lie algebra $\mathfrak{S D}(2,2)$ of the Special pseudo-orthogonal group $S O(2,2)$ i.e $\exp (\mathcal{X}) \in S O(2,2)$. It follows from the relations (1) (2) and (3) that the special pseudo- orthogonal transformation defined by $\exp (\mathcal{X})$ is associated with the LCT $g$ defined by
(1) and (2). This correspondence defines a representation of the LCT group with special pseudo-orthogonal transformations on the operator space $\mathbb{E}=\left\{\left(\begin{array}{llll}\not^{+} & \mathscr{X}^{-} & \mathscr{X}^{+} & \not^{-}\end{array}\right)\right\}$.

\subsection{Case of N-Dime Nsional Theory}

As in our work [2], we consider the case of a general linear canonical transformation

$$
\left\{\begin{array}{l}
\not_{\mu}{ }^{\prime}=\Pi_{\mu}^{v} \not_{v}+\Theta_{\mu}^{v} \mathscr{X}_{v} \\
\mathscr{X}_{\mu}{ }^{\prime}=\Xi_{\mu}^{v} \not_{v}+\Lambda_{\mu}^{v} \mathfrak{X}_{v}
\end{array} \Leftrightarrow\left(\begin{array}{ll}
p^{\prime} & \mathscr{X}^{\prime}
\end{array}\right)=\left(\begin{array}{ll}
\not & \mathscr{X}
\end{array}\right)\left(\begin{array}{cc}
\Pi & \Xi \\
\Theta & \Lambda
\end{array}\right)\right.
$$

which leaves invariant the canonical commutation relations

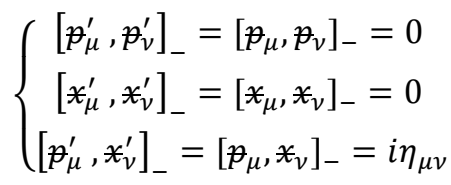

In these expressions, we have $\mu=0, \ldots N-1$. $p$ and $x$ are the $1 \times N$ row matrices (covectors) with components $\beta_{\mu}$ and $*_{\mu} . \Pi, \Xi, \Theta$ and $\Lambda$ are $N \times N$ square matrices and $\eta_{\mu \nu}$ are the covariant components of the bilinear form with a signature $\left(N_{+}, N_{-}\right)$with $N_{+}+N_{-}=N$. Following our paper [2], the $2 N \times 2 N$ matrix $g=\left(\begin{array}{cc}\Pi & \Xi \\ \Theta & \Lambda\end{array}\right)$ belongs to the pseudosymplectic group $S p\left(2 N_{+}, 2 N_{-}\right) \cdot g$ can be written in the form

$$
g=\left(\begin{array}{ll}
\Pi & \Xi \\
\Theta & \Lambda
\end{array}\right)=\exp \left[\left(\begin{array}{ll}
\lambda+\mu & \varphi-\theta \\
\varphi+\theta & \lambda-\mu
\end{array}\right)\right]
$$

in which $\theta, \varphi, \mu$ and $\lambda$ are $N \times N$ matrices which satisfy the following relations

$$
\left\{\begin{array}{c}
\theta^{T}=\eta \theta \eta \\
\varphi^{T}=\eta \varphi \eta \\
\mu^{T}=\eta \mu \eta \\
\lambda^{T}=-\eta \lambda \eta \text { and } \operatorname{Tr}(\lambda)=0
\end{array}\right.
$$

The relation (9) is the multidimensional generalization of the parameterization (2). To associate a special pseudoorthogonal transformation to the LCT defined by $g$, as generalization of the relations (3), we introduce the operators

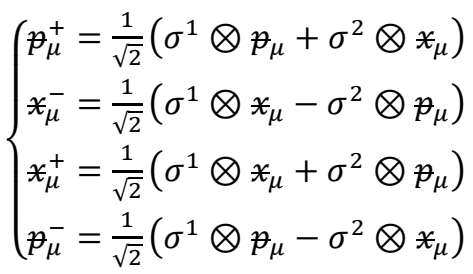

If we denote $p^{+}, x^{-}, x^{+}, p^{-}$the $1 \times N$ row matrices

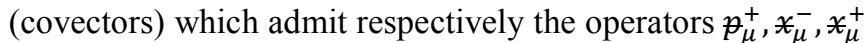
and $p_{\mu}^{-}$as components, we can deduce from the relations (7), (9) and (11) that for an infinitesimal LCT, we have 


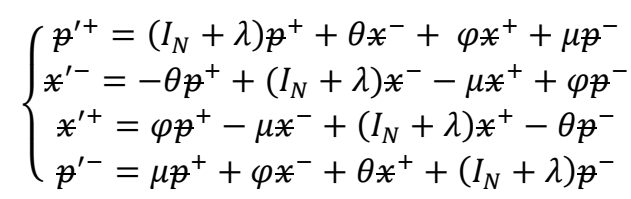

in which $I_{N}$ is the $N \times N$ identity matrix. The infinitesimal transformation (12) can be put in the matricial form

$$
\left(\begin{array}{llll}
\not^{+} & x^{\prime-} & x^{\prime+} & p^{\prime-}
\end{array}\right)=\left(\begin{array}{llll}
\not^{+} & \mathscr{X}^{-} & x^{+} & p^{-}
\end{array}\right)\left(I_{4 N}+X\right)
$$

in which $I_{4 N}$ is the $4 N \times 4 N$ identity matrix and $\mathcal{X}$ is the $4 N \times 4 N$ square matrix

$$
\mathcal{X}=\left(\begin{array}{cccc}
\lambda & -\theta & \varphi & \mu \\
\theta & \lambda & -\mu & \varphi \\
\varphi & -\mu & \lambda & \theta \\
\mu & \varphi & -\theta & \lambda
\end{array}\right)
$$

The matrix $\mathcal{X}$ belongs to the Lie algebra $\mathfrak{s D}(2 N, 2 N)$ of the Special pseudo-Orthogonal group $S O(2 N, 2 N)$ i.e $e^{x} \in$ $S O(2 N, 2 N)$. The relation (11) then leads to a correspondence between the LCT $g$ defined by (7) and (9) and the special pseudo- orthogonal transformation defined by $\exp (\mathcal{X})$.

\section{Spinorial Representation of a LCT}

\subsection{Definition of the Spinorial Representation}

As established in the previous section, any LCT can be associated with a special pseudo-orthogonal transformation.
But any special pseudo-orthogonal transformation has a spinorial representation (a spin group is a double cover of a special orthogonal group). It follows that we can construct a spinorial representation of an LCT too. To do so, we introduce the operator

$$
\mathbb{P}=\left(\alpha_{+} \otimes p^{+}+\beta_{+} \otimes \mathscr{X}^{-}+\beta_{-} \otimes \mathfrak{X}^{+}+\alpha_{-} \otimes p^{-}\right)
$$

in which the operators ${\not^{+}}^{+} \mathscr{X}^{-}, \mathfrak{X}^{+}$and $\not^{-}$are the operator defined in the relation (3) and $\alpha_{+}, \beta_{+}, \beta_{-}, \alpha_{-}$are the generators of the Clifford algebra $\mathfrak{C}(2,2)$. They satisfy the relations

$$
\left\{\begin{array}{c}
\left(\alpha_{+}\right)^{2}=\left(\beta_{+}\right)^{2}=\mathbb{I} \\
\left(\beta_{-}\right)^{2}=\left(\alpha_{-}\right)^{2}=-\mathbb{I} \\
\alpha_{+} \beta_{+}+\beta_{+} \alpha_{+}=0 \\
\alpha_{+} \beta_{-}+\beta_{-} \alpha_{+}=0 \\
\alpha_{+} \alpha_{-}+\alpha_{-} \alpha_{+}=0 \\
\beta_{+} \alpha_{-}+\alpha_{-} \beta_{+}=0 \\
\beta_{+} \beta_{-}+\beta_{-} \beta_{+}=0 \\
\beta_{-} \alpha_{-}+\alpha_{-} \beta_{-}=0
\end{array}\right.
$$

where II is the identity in the Clifford algebra $\mathfrak{C}(2.2)$. From the relation (16), it can be established that the operators $\alpha_{+}, \beta_{+}, \beta_{-}, \alpha_{-}$satisfy also the following commutations relations.

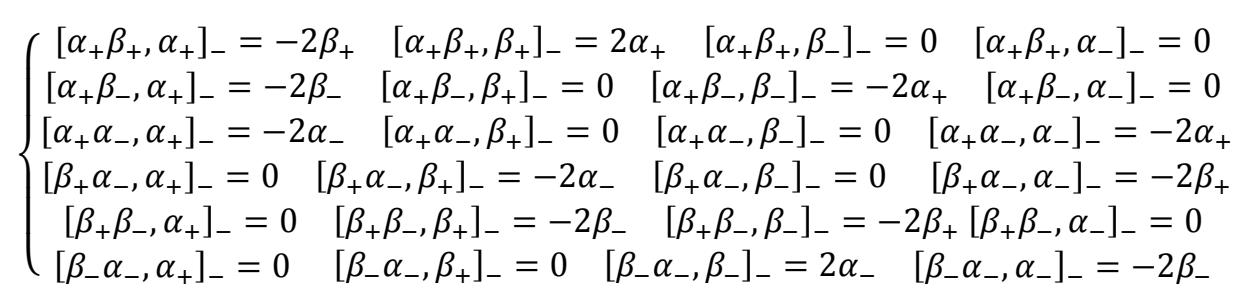

Let us denote $\operatorname{Spin}(2,2) \otimes I_{2}=\left\{\mathcal{S}=\exp (\vartheta) \otimes I_{2}\right\}$ in which $\exp (\vartheta)$ is an element of the group $\operatorname{Spin}(2,2)$ and $I_{2}$ the $2 \times 2$

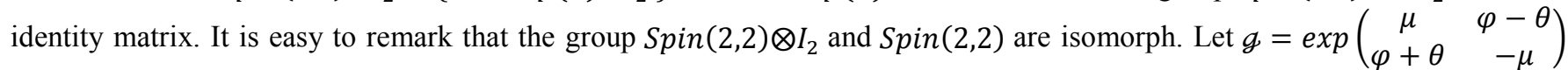
be the element of the LCT group $S L(2)$ corresponding to an LCT (15) and $\mathcal{S}$ the element of $\operatorname{Spin}(2,2) \otimes I_{2}$ associated with $g$. This correspondence between $g$ and $\mathcal{S}$ can be described with a mapping $\varrho$ between the LCT group $S L(2)$ and $n(2,2) \otimes I_{2}$.

$$
\mathcal{S}=\varrho(g) \Leftrightarrow\left\{\begin{array}{c}
\left(\begin{array}{ll}
\mathcal{P}^{\prime} & \mathfrak{X}^{\prime}
\end{array}\right)=\left(\begin{array}{cc}
p & x
\end{array}\right) g=\left(\begin{array}{ll}
p & x
\end{array}\right) \exp \left(\begin{array}{cc}
\mu & \varphi-\theta \\
\varphi+\theta & -\mu
\end{array}\right) \\
\mathbb{P}^{\prime}=\mathcal{S}_{\mathbb{P} \mathcal{S}^{-1}}
\end{array}\right.
$$

$\mathcal{S}$ can be put in the form

$$
\mathcal{S}=\exp \left(\vartheta \otimes I_{2}\right)=\exp \left[\left(\vartheta^{1} \alpha_{+} \beta_{+}+\vartheta^{2} \alpha_{+} \beta_{-}+\vartheta^{3} \alpha_{+} \alpha_{-}+\vartheta^{4} \beta_{+} \beta_{-}+\vartheta^{5} \beta_{+} \alpha_{-}+\vartheta^{6} \beta_{-} \alpha_{-}\right) \otimes I_{2}\right]
$$

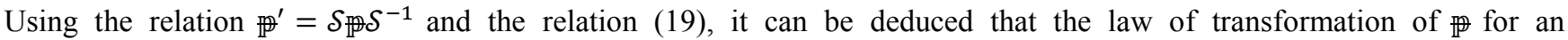
infinitesimal linear canonical transformation is

$$
\mathbb{P}^{\prime}=\mathbb{P}+\left[\vartheta \otimes I_{2}, \mathbb{P}\right]_{-}
$$

Taking into account the expression (15) of $p$ and the relations (17) and (20), we deduce the relations 


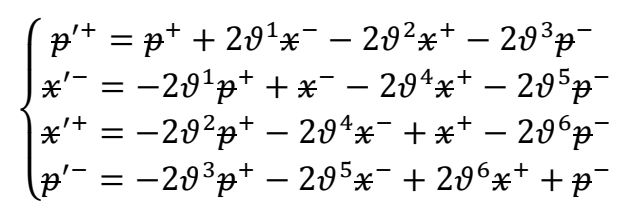

The identification of the relation (21) with (4) permits to deduce the expressions of $\vartheta^{1}, \vartheta^{2}, \vartheta^{3}, \vartheta^{4}, \vartheta^{5}$ and $\vartheta^{6}$ in terms of $\theta, \varphi$ and $\mu$

$$
\left\{\begin{array}{lll}
\vartheta^{1}=\frac{\theta}{2} & \vartheta^{2}=-\frac{\varphi}{2} & \vartheta^{3}=-\frac{\mu}{2} \\
\vartheta^{4}=\frac{\mu}{2} & \vartheta^{5}=-\frac{\varphi}{2} & \vartheta^{6}=\frac{\theta}{2}
\end{array}\right.
$$

The expression (19) of $\mathcal{S}$ becomes

$$
\mathcal{S}=\exp \left(\vartheta \otimes I_{2}\right)=\exp \left\{\left[\frac{\theta}{2}\left(\alpha_{+} \beta_{+}+\beta_{-} \alpha_{-}\right)-\frac{\varphi}{2}\left(\alpha_{+} \beta_{-}+\beta_{+} \alpha_{-}\right)-\frac{\mu}{2}\left(\alpha_{+} \alpha_{-}-\beta_{+} \beta_{-}\right)\right] \otimes I_{2}\right\}
$$

The operator $\mathcal{S}$ can be considered as acting on the element $\vec{\psi}$ of a spinor space $\mathbb{S}$

$$
\vec{\psi}^{\prime}=\mathcal{S}(\vec{\psi}) \Leftrightarrow \psi^{\prime a}=\mathcal{S}_{b}^{a} \psi^{b}
$$

The couple $(\mathbb{S}, \varrho$ ), in which $\varrho$ is the mapping in $(18)$, then define a spinorial representation of the LCT group.

\subsection{Expression of the Invariant as Polynomial of the Operator $\mathbb{P}$}

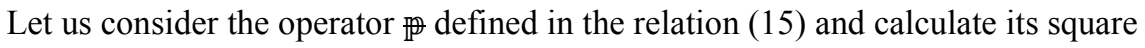

$$
(\mathbb{P})^{2}=\left(\alpha_{+} \otimes \mathfrak{p}^{+}+\beta_{+} \otimes \mathscr{*}^{-}+\beta_{-} \otimes \mathscr{*}^{+}+\alpha_{-} \otimes{p^{-}}^{2}\right.
$$

From the expression (3) of $p^{+}, x^{-}, x^{+}, p^{-}$, we deduce the relations

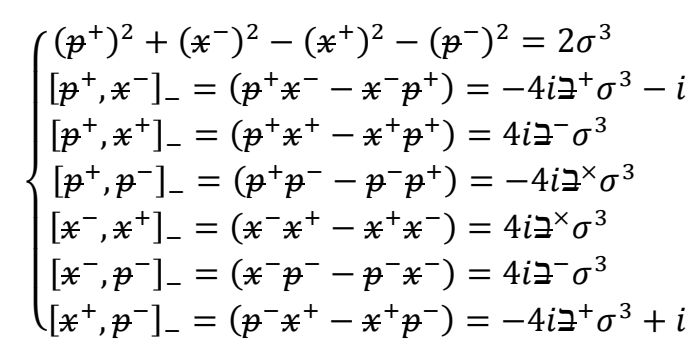

in which $\beth^{+}, \beth^{-}$and $\beth^{\times}$are the reduced dispersion operators [2]

$$
\left\{\begin{array}{l}
\beth^{+}=\frac{1}{4}\left[(\not{p})^{2}+(\not{x})^{2}\right] \\
\beth^{-}=\frac{1}{4}\left[(\not{p})^{2}-(\not{x})^{2}\right] \\
\beth^{\times}=\frac{1}{4}[\not \not x+\not p]
\end{array}\right.
$$

Using the relations (16), (26) and (27), we can deduce the expression of $(\mathbb{P})^{2}$

$$
(\mathbb{P})^{2}=\mathbb{B}+2 \mathbb{I} \otimes \sigma^{3}-i\left(\alpha_{+} \beta_{+}-\beta_{-} \alpha_{-}\right) \otimes I_{2}
$$

in which $\mathbb{B}$ is the operator

$$
\mathbb{B}=-4 i\left[\left(\alpha_{+} \beta_{+}+\beta_{-} \alpha_{-}\right) \otimes \sigma^{3} \otimes \beth^{+}-\left(\alpha_{+} \beta_{-}+\beta_{+} \alpha_{-}\right) \otimes \sigma^{3} \otimes \beth^{-}+\left(\alpha_{+} \alpha_{-}-\beta_{+} \beta_{-}\right) \otimes \sigma^{3} \otimes \beth^{\times}\right]
$$

$\mathbb{I}$ is the identity operator in the Clifford algebra $\mathfrak{C}(2.2)$ and $I_{2}$ is $2 \times 2$ identity matrix. If we introduce the operators 


$$
\left\{\begin{array}{l}
\mho_{+}=\frac{1}{2}\left(\alpha_{+} \beta_{+}+\beta_{-} \alpha_{-}\right) \otimes \sigma^{3} \\
\mho_{-}=\frac{1}{2}\left(\alpha_{+} \beta_{-}+\beta_{+} \alpha_{-}\right) \otimes \sigma^{3} \\
\mho_{\times}=\frac{1}{2}\left(\alpha_{+} \alpha_{-}-\beta_{+} \beta_{-}\right) \otimes \sigma^{3} \\
\mho_{o}=\frac{1}{2}\left(\alpha_{+} \beta_{+}-\beta_{-} \alpha_{-}\right) \otimes \sigma^{3}
\end{array}\right.
$$

we obtain as expression of

$$
\mathbb{D}=-8 i\left[\mho_{+} \otimes \beth^{+}-\mho_{-} \otimes \beth^{-}+\mho_{\times} \otimes \beth^{\times}\right]
$$

using the fact that $\left(\sigma^{3}\right)^{2}=I_{2}$, we may write also for the expression (29) of ( $\left.\mathbb{P}\right)^{2}$ and the expression (23) of $\mathcal{S}$

$$
\begin{gathered}
(\mathbb{P})^{2}=\mathbb{W}+2\left(\mathbb{I} \otimes \sigma^{3}\right)\left(\mathbb{I} \otimes I_{2}-i \mho_{o}\right) \\
\mathcal{S}=\exp \left(\vartheta \otimes I_{2}\right)=\exp \left[\left(\theta \mho_{+}-\varphi \mho_{-}-\mu \mho_{\times}\right)\left(\mathbb{I} \otimes \sigma^{3}\right)\right]
\end{gathered}
$$

It can be deduced from the relations (16) and (30) that the operators $\mho_{+}, \mho_{-}, \mho_{\times}$and $\mho_{o}$ satisfy the relations

$$
\left\{\begin{array}{l}
\left(\mho_{+}\right)^{2}=\frac{1}{2}(\epsilon-\mathbb{I}) \otimes I_{2} \\
\left(\mho_{-}\right)^{2}=-\frac{1}{2}(\epsilon-\mathbb{I}) \otimes I_{2} \\
\left(\mho_{\times}\right)^{2}=-\frac{1}{2}(\epsilon-\mathbb{I}) \otimes I_{2} \\
\left(\mho_{o}\right)^{2}=-\frac{1}{2}(\epsilon+\mathbb{I}) \otimes I_{2} \\
\mho_{+} \mho_{-}=-\mho_{-} \mho_{+}=\left(\mathbb{I} \otimes \sigma^{3}\right) \mho_{\times} \\
\mho_{-} \mho_{\times}=-\mho_{\times} \mho_{-}=-\left(\mathbb{I} \otimes \sigma^{3}\right) \mho_{+} \\
\mho_{\times} \mho_{+}=-\mho_{+} \mho_{\times}=\left(\mathbb{I} \otimes \sigma^{3}\right) \mho_{-} \\
\mho_{+} \mho_{o}=\mho_{o} \mho_{+}=0 \\
\mho_{-} \mho_{o}=\mho_{o} \mho_{-}=0 \\
\mho_{+} \mho_{o}=\mho_{o} \mho_{+}=0
\end{array}\right.
$$

in which

$$
\epsilon=\alpha_{+} \beta_{+} \beta_{-} \alpha_{-}
$$

According to the relation (19), the law of transformation of $\mathbb{P}$ is $\mathbb{P}^{\prime}=\mathcal{S} \mathbb{P} \mathcal{S}^{-1}$. It follows that we have for the law of transformation of $(\mathbb{P})^{2}$

$$
\left(\mathbb{P}^{\prime}\right)^{2}=\mathbb{P}^{\prime} \mathbb{P}^{\prime}=\mathcal{S} \mathbb{P} \mathcal{S}^{-1} \mathcal{S} \mathbb{P} \mathcal{S}^{-1}=\mathcal{S}(\mathbb{P})^{2} \mathcal{S}^{-1}=\mathcal{S} \mathbb{D} \mathcal{S}^{-1}+\left(2 \mathbb{I} \otimes \sigma^{3}\right)\left(\mathbb{I} \otimes I_{2}-i \mho_{o}\right)
$$

as we have $\delta \mathbb{P} \mathcal{S}^{-1} \neq \mathbb{D}$, it follows that $\left(\mathbb{P}^{\prime}\right)^{2} \neq(\mathbb{P})^{2}$ i.e $(\mathbb{P})^{2}$ is not an invariant. However, it can be shown that an invariant is the polynomial of $4^{\text {th }}$ degree $(\mathbb{P})^{4}+4\left(\mathbb{I} \otimes \sigma^{3}\right)(\mathbb{P})^{2}$ i.e we have the relation

$$
\left(\mathbb{P}^{\prime}\right)^{4}+4\left(\mathbb{I} \otimes \sigma^{3}\right)\left(\mathbb{P}^{\prime}\right)^{2}=\mathcal{S}\left[(\mathbb{P})^{4}+4\left(\mathbb{I} \otimes \sigma^{3}\right)(\mathbb{P})^{2}\right] \mathcal{S}^{-1}=(\mathbb{P})^{4}+4\left(\mathbb{I} \otimes \sigma^{3}\right)(\mathbb{P})^{2}
$$

\subsection{Case of Multidimensional Theory}

According to the relations (12), (13) and (14), the special pseudo-orthogonal transformation corresponding to the LCT defined by the relations (7), (8) and (9) is an element of the group $S O(2 N, 2 N)$ so the spin group which is to be used to construct the spinorial representation is $\operatorname{Spin}(2 N, 2 N)$. Generalizing the relation (15), we introduce

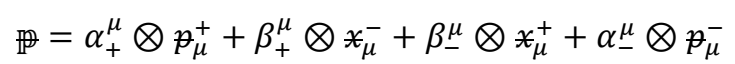

in which $\mathcal{p}_{\mu}^{+}, \mathscr{\varkappa}_{\mu}^{-}, \mathfrak{x}_{\mu}^{+}$and $\mathcal{p}_{\mu}^{-}$are the operators defined in the relation (11) and the $4 N$ operators $\alpha_{+}^{\mu}, \beta_{+}^{\mu}, \beta_{-}^{\mu}, \alpha_{-}^{\mu}(\mu=0,1, \ldots, N-$ 1) are the generators of the Clifford algebra $\mathfrak{C}(2 N, 2 N)$. They are characterized by the relations. 


$$
\begin{cases}\alpha_{+}^{\mu} \alpha_{+}^{v}+\alpha_{+}^{v} \alpha_{+}^{\mu}=2 \eta^{\mu v} \mathbb{I} & \beta_{+}^{\mu} \beta_{+}^{v}+\beta_{+}^{v} \beta_{+}^{\mu}=2 \eta^{\mu v} \mathbb{I} \\ \beta_{-}^{\mu} \beta_{-}^{v}+\beta_{-}^{v} \beta_{-}^{\mu}=-2 \eta^{\mu \nu} \mathbb{I} & \alpha_{-}^{\mu} \alpha_{-}^{v}+\alpha_{-}^{v} \alpha_{-}^{\mu}=-2 \eta^{\mu v} \mathbb{I} \\ \alpha_{+}^{\mu} \beta_{+}^{v}+\beta_{+}^{v} \alpha_{+}^{\mu}=0 & \alpha_{+}^{\mu} \beta_{-}^{v}+\beta_{-}^{v} \alpha_{+}^{\mu}=0 \\ \alpha_{+}^{\mu} \alpha_{-}^{v}+\alpha_{-}^{v} \alpha_{+}^{\mu}=0 & \beta_{+}^{\mu} \beta_{-}^{v}+\beta_{-}^{v} \beta_{+}^{\mu}=0 \\ \beta_{+}^{\mu} \alpha_{-}^{v}+\alpha_{-}^{v} \beta_{+}^{\mu}=0 & \beta_{-}^{\mu} \alpha_{-}^{v}+\alpha_{-}^{v} \beta_{-}^{\mu}=0\end{cases}
$$

in which $\mathbb{I}$ is the identity of the Clifford algebra $\mathbb{C}(2 N, 2 N)$. It can be established that the operators $\alpha_{+}^{\mu}, \beta_{+}^{\mu}, \beta_{-}^{\mu}, \alpha_{-}^{\mu}$ satisfy the following commutations relations

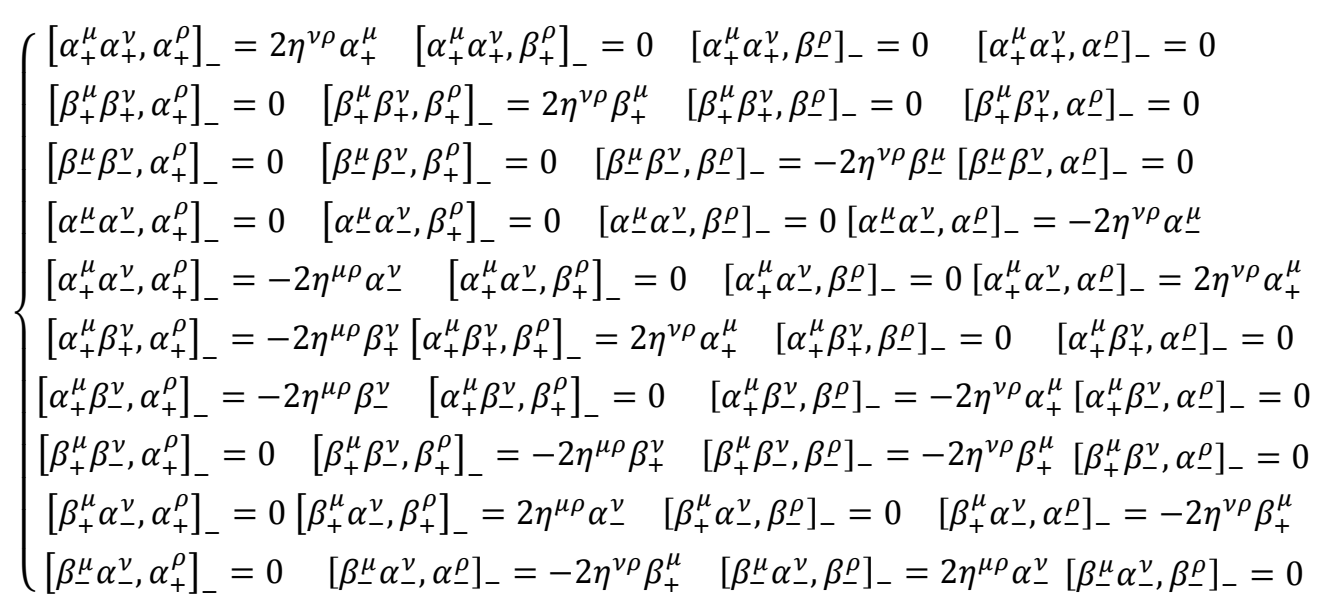

Let us consider the set

$$
\operatorname{Spin}(2 N, 2 N) \otimes I_{2}=\left\{\mathcal{S}=\exp (\vartheta) \otimes I_{2}=\exp \left(\vartheta \otimes I_{2}\right)\right\}
$$

in which $\exp (\vartheta)$ is an element of $\operatorname{Spin}(2 N, 2 N)$ and $I_{2}$ the $2 \times 2$ identity matrix. Let $g=\exp \left[\left(\begin{array}{cc}\lambda+\mu & \varphi-\theta \\ \varphi+\theta & \lambda-\mu\end{array}\right)\right]$ be the element of the LCT group $\operatorname{Sp}\left(2 N_{+}, 2 N_{-}\right)$corresponding to an LCT (21) and $\mathcal{S}$ the element of $\operatorname{Spin}(2 N, 2 N) \otimes I_{2}$ associated with $g$. The correspondence between $g$ and $\delta$ can be described with a mapping $\varrho$ between the LCT group $S p\left(2 N_{+}, 2 N_{-}\right)$and $\operatorname{Spin}(2 N, 2 N) \otimes I_{2}$.

$$
\mathcal{S}=\varrho(g) \Leftrightarrow\left\{\begin{array}{c}
\left(p^{\prime} \quad \mathcal{X}^{\prime}\right)=\left(\begin{array}{cc}
p & x
\end{array}\right) g=\left(\begin{array}{ll}
p & x
\end{array}\right) \exp \left[\left(\begin{array}{cc}
\lambda+\mu & \varphi-\theta \\
\varphi+\theta & \lambda-\mu
\end{array}\right)\right] \\
\mathbb{P}^{\prime}=\mathcal{S} \mathbb{P} \mathcal{S}^{-1}
\end{array}\right.
$$

For an infinitesimal LCT, the law of transformation of $p$ is

$$
\mathbb{P}^{\prime}=\mathbb{P}+\left[\vartheta \otimes I_{2}, \mathbb{P}\right]_{-}
$$

Using the fact that $\vartheta$ belongs to the Lie algebra of $\operatorname{Spin}(2 N, 2 N)$ and taking into account the relations (11), (12) (38), (40) and (42) we can find the following expression for $\vartheta$

$$
\vartheta=\eta_{v \rho}\left[\frac{\theta_{\mu}^{\rho}}{2}\left(\alpha_{+}^{\mu} \beta_{+}^{v}+\beta_{-}^{v} \alpha_{-}^{\mu}\right)-\frac{\varphi_{\mu}^{\rho}}{2}\left(\alpha_{+}^{\mu} \beta_{-}^{v}+\beta_{+}^{v} \alpha_{-}^{\mu}\right)+\frac{\mu_{\mu}^{\rho}}{2}\left(\alpha_{+}^{\mu} \alpha_{-}^{v}+\beta_{+}^{v} \beta_{-}^{\mu}\right)+\frac{\lambda_{\mu}^{\rho}}{2}\left(\alpha_{+}^{\mu} \alpha_{+}^{v}+\beta_{+}^{\mu} \beta_{+}^{v}-\beta_{-}^{\mu} \beta_{-}^{v}-\alpha_{-}^{\mu} \alpha_{-}^{v}\right)\right]
$$

then we have for $\mathcal{S}$

$$
\mathcal{S}=\exp \left\{\eta_{v \rho}\left[\frac{\theta_{\mu}^{\rho}}{2}\left(\alpha_{+}^{\mu} \beta_{+}^{\nu}+\beta_{-}^{v} \alpha_{-}^{\mu}\right)-\frac{\varphi_{\mu}^{\rho}}{2}\left(\alpha_{+}^{\mu} \beta_{-}^{\nu}+\beta_{+}^{\nu} \alpha_{-}^{\mu}\right)+\frac{\mu_{\mu}^{\rho}}{2}\left(\alpha_{+}^{\mu} \alpha_{-}^{v}+\beta_{+}^{\nu} \beta_{-}^{\mu}\right)+\frac{\lambda_{\mu}^{\rho}}{2}\left(\alpha_{+}^{\mu} \alpha_{+}^{v}+\beta_{+}^{\mu} \beta_{+}^{\nu}-\beta_{-}^{\mu} \beta_{-}^{\nu}-\alpha_{-}^{\mu} \alpha_{-}^{v}\right)\right] \otimes I_{2}\right\}
$$

The operator $\mathcal{S}$ can be considered as acting on the spinors $\vec{\psi}$ belonging to a spinor space $\mathbb{S}$

$$
\vec{\psi}^{\prime}=\delta \vec{\psi} \Leftrightarrow \psi^{\prime a}=\mathcal{S}_{b}^{a} \psi^{b}
$$

The couple $(\mathbb{S}, \varrho)$ define a spinorial representation of the LCT group.

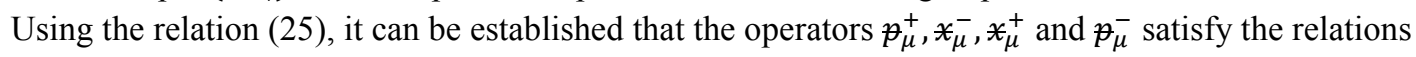




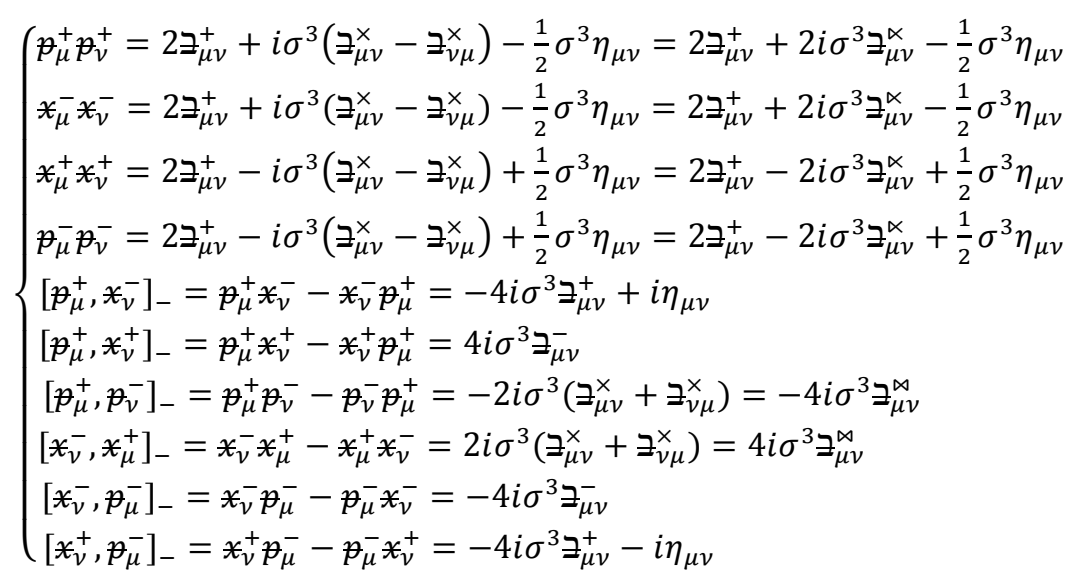

in which [2]

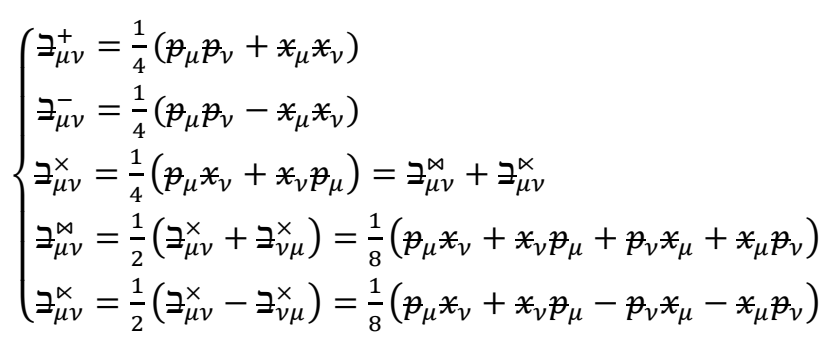

From the relations (46) and (47), we have for the square $(\mathbb{P})^{2}$ of the operator $¥$

$$
(\mathbb{P})^{2}=\left(\alpha_{+}^{\mu} \otimes p_{\mu}^{+}+\beta_{+}^{\mu} \otimes x_{\mu}^{-}+\beta_{-}^{\mu} \otimes x_{\mu}^{+}+\alpha_{-}^{\mu} \otimes p_{\mu}^{-}\right)^{2}=\mathbb{D}-2\left(\mathbb{I} \otimes \sigma^{3}\right)\left(N \mathbb{I} \otimes I_{2}-i \eta_{\mu \nu} \mho_{o}^{\mu \nu}\right)
$$

in which

$$
\begin{aligned}
& \mathbb{B}=-8 i\left[\mho_{+}^{\mu \nu} \otimes \beth_{\mu \nu}^{+}-\mho_{-}^{\mu \nu} \otimes \beth_{\mu \nu}^{-}+\mho_{\bowtie}^{\mu \nu} \otimes \beth_{\mu \nu}^{\infty}-\beth_{\mu \nu}^{\propto} \otimes \mho_{\ltimes}^{\mu \nu}\right] \\
& \left\{\begin{array}{l}
\mho_{+}^{\mu \nu}=\frac{1}{2}\left[\left(\alpha_{+}^{\mu} \beta_{+}^{v}+\beta_{-}^{v} \alpha_{-}^{\mu}\right) \otimes \sigma^{3}\right] \\
\mho_{-}^{\mu \nu}=\frac{1}{2}\left[\left(\alpha_{+}^{\mu} \beta_{-}^{v}+\beta_{+}^{v} \alpha_{-}^{\mu}\right) \otimes \sigma^{3}\right] \\
\mho_{\bowtie}^{\mu \nu}=\frac{1}{2}\left[\left(\alpha_{+}^{\mu} \alpha_{-}^{v}+\beta_{+}^{v} \beta_{-}^{\mu}\right) \otimes \sigma^{3}\right] \\
\mho_{\ltimes}^{\mu \nu}=\frac{1}{4}\left[\left(\alpha_{+}^{\mu} \alpha_{+}^{v}+\beta_{+}^{\mu} \beta_{+}^{v}-\beta_{-}^{\mu} \beta_{-}^{\nu}-\alpha_{-}^{\mu} \alpha_{-}^{\nu}\right) \otimes \sigma^{3}\right] \\
\mho_{o}^{\mu \nu}=\frac{1}{2}\left[\left(\alpha_{+}^{\mu} \beta_{+}^{v}-\beta_{-}^{v} \alpha_{-}^{\mu}\right) \otimes \sigma^{3}\right]
\end{array}\right.
\end{aligned}
$$

Using the relation (50), the expression of the operator $\mathcal{S}$ in (44) may be written in a compact form

$$
\mathcal{S}=\exp \left[\eta_{\nu \rho}\left(\theta_{\mu}^{\rho} \mho_{+}^{\mu \nu}-\varphi_{\mu}^{\rho} \mho_{+}^{\mu \nu}+\mu_{\mu}^{\rho} \mho_{\bowtie}^{\mu \nu}+\lambda_{\mu}^{\rho} \mho_{\ltimes}^{\mu \nu}\right)\left(\mathbb{I} \otimes \sigma^{3}\right)\right]
$$

The operator $\mathbb{D}$ defined by the relation (49) generalizes the one defined, for one dimension, in (29), (31). It is obvious to note that the operators $(\mathbb{P})^{2}$ and $\mathbb{W}$ are not invariant. The case of one dimension theory, studied in the section 3.2, suggests that an invariant may be polynomial function in with a degree greater than or equal to 4 .

\section{Conclusion}

The approach described in this work shows that it is possible to establish a spinorial representation of Linear Canonical Transformations. As it is shown in the sections 2 and 3, the establishment of this spinorial representation follows from the fact that, using an adequate parameterization, it is possible to associate with a linear canonical transformation a special pseudo-orthogonal transformation in an operator space. Then the spinorial representation can be established easily using the relations between special pseudo-orthogonal group and spin group.

A main result thus obtained is the explicit expression of the operator $\mathcal{S}$ which corresponds to the representation of an LCT in a spinor space. It is given in the relation (23) or (33) for the case of one dimension theory and in the relation (44) or (51) for the multidimensional case.

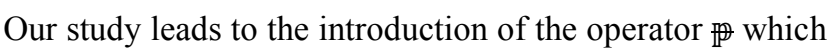


is given respectively for one dimension theory and multidimensional cases in the relations (15) and (38). As shown by the relations (18) and (41), this operator is useful to write the explicit expression of the transformation corresponding to the spinorial representation of the LCT. According to the relation (37), the invariant is not $(\mathbb{P})^{2}$, as it may be expected for a spinorial representation associated to an ordinary commutative vector space, but a higher degree

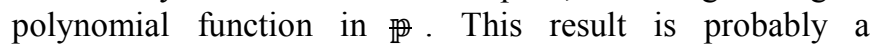
consequence of the fact that the space on which the LCT is defined is a noncommutative operator space.

According to our works [14] and [15], LCTs can be linked with many interesting physical problems like the generalization of Fourier and Lorentz transformations in the framework of a relativistic quantum theory and the study of the properties of elementary fermions of the Standard Model. Following this way, we have, for instance, established in [15] a new approach to explain the charges (electric charge, hypercharge and colors) of the elementary fermions. It was shown that the electric charge can be written as the sum of four terms, the weak hypercharge of five terms and the weak isospin of two terms. These facts suggest us to expect that the results established in the present paper may have many interesting application in quantum theory and related domains.

\section{References}

[1] Raoelina Andriambololona, Ravo Tokiniaina Ranaivoson, Rakotoson Hanitriarivo, Wilfrid Chrysante Solofoarisina, "Study on Linear Canonical Transformation in a Framework of a Phase Space Representation of Quantum Mechanics", arXiv: 1503.02449 [quant-ph], International Journal of Applied Mathematics and Theoretical Physics. Vol. 1, No. 1, 2015, pp. 1-8, 2015.

[2] Raoelina Andriambololona, Ravo Tokiniaina Ranaivoson, Rakotoson Hanitriarivo, Damo Emile Randriamisy, "Dispersion Operator Algebra and Linear Canonical Transformation", arXiv: 1608.02268v2 [quant-ph], International Journal of Theoretical Physics, Volume 56, Issue 4, pp 1258-1273, Springer, April 2017.

[3] Ravo Tokiniaina Ranaivoson, Raoelina Andriambololona, Rakotoson Hanitriarivo, Roland Raboanary: "Study on a Phase Space Representation of Quantum Theory", arXiv: 1304.1034 [quant-ph], International Journal of Latest Research in Science and Technology, ISSN(Online): 22785299, Volume 2,Issue 2: Page No.26-35, March-April, 2013.
[4] Rakotoson Hanitriarivo, Raoelina Andriambololona, Ravo Tokiniaina Ranaivoson, Raboanary Roland: "Coordinate, momentum and dispersion operators in phase space representation", arXiv: 1707.02223 [quant-ph], International Journal of Latest Research in Science and Technology ISSN (Online): 2278-5299 Volume 6, Issue 4: Page No. 8-13, JulyAugust 2017.

[5] Tatiana Alieva, Martin J. Bastiaans, "The Linear Canonical Transformations: Definition and Properties", In: Healy J., Alper Kutay M., Ozaktas H., Sheridan J. (eds) Linear Canonical Transforms. Springer Series in Optical Sciences, vol 198. Springer, New York, 2016.

[6] Tian-Zhou Xu, Bing-Zhao Li: Linear Canonical Transform and Its Applications, Science Press, Beijing, China, 2013.

[7] M. Moshinsky and C. Quesne: Linear canonical transformations and their unitary representations, J. Math. Phys.12, 8, 1772-1783, 1971.

[8] K. B. Wolf: A Top-Down Account of Linear Canonical Transforms, arXiv: 1206.1123 [Math.ph], SIGMA 8 (2012), 033, 13 pages.

[9] J. J. Healy, M. A. Kutay, H. M. Ozaktas and J. T. Sheridan, "Linear Canonical Transforms: Theory and Applications", Springer, New York, 2016.

[10] Robert Coquereaux, Espaces fibrés et connexions, Une introduction aux géométries classiques et quantiques de la physique théorique,Centre de Physique Théorique, LuminyMarseille, 2002.

[11] Robert Coquereaux, Clifford algebras, spinors and fundamental interactions: Twenty Years After, arXiv: mathph/0509040, 2005.

[12] I. Todorov, Clifford Algebras and Spinors, Bulg. J. Phys. 38 (2011) 3-28.

[13] Raoelina Andriambololona: Algèbre linéaire et multilinéaire, Collection LIRA, INSTN- Madagascar, 1986.

[14] Rakotoson Hanitriarivo, Raoelina Andriambololona, Ravo Tokiniaina Ranaivoson, Roland Raboanary, Linear Canonical Transformations in Relativistic Quantum Physics, arXiv: 1804.10053 [quant-ph], 2018.

[15] Ravo Tokiniaina Ranaivoson, Raoelina Andriambololona, Rakotoson Hanitriarivo, Properties of Elementary Fermions of the Standard Model deduced from Linear Canonical Transformations, arXiv: 1806.07228 [physics.gen-ph], 2018. 